\title{
Völlig unterschiedlich oder doch recht ähnlich? Die soziodemografische Struktur der AOK Niedersachsen im Vergleich zur niedersächsischen und bundesweiten Allgemein- und Erwerbsbevölkerung
}

\author{
Completely Different or Quite Similar? The Sociodemographic \\ Structure of the AOK Lower Saxony in Comparison to the General \\ and Working Population in Lower Saxony and the Federal \\ Republic of Germany
}

\author{
Autoren \\ Jelena Epping1, Siegfried Geyer ${ }^{1}$, Sveja Eberhard², Juliane Tetzlaff1
}

Institute

1 Medizinische Soziologie, Medizinische Hochschule Hannover, Hannover, Deutschland

2 Stabsbereich Politik, Forschung \& Presse, Allgemeine Ortskrankenkasse Niedersachsen, Hannover, Deutschland

\section{Schlüsselwörter}

Krankenkassendaten, soziodemographische Struktur, Repräsentativität, Erwerbsbevölkerung, Tätigkeitskomplexität

\section{Keywords}

health insurance claims data, sociodemographic structure, representativeness, occupation, education

online publiziert $\quad 25.10 .2021$

\author{
Bibliografie \\ DOI 10.1055/a-1553-3565 \\ ISSN 0949-7013 \\ (c) 2021. Thieme. All rights reserved. \\ Georg Thieme Verlag, Rüdigerstraße 14, \\ 70469 Stuttgart, Germany \\ Korrespondenzadresse \\ Jelena Epping \\ Medizinische Soziologie \\ Medizinische Hochschule Hannover \\ Carl-Neuberg-Straße 1 \\ 30625 Hannover \\ Deutschland \\ epping.jelena@mh-hannover.de
}

Gesundheitswesen 2021; 83 (Suppl. 2): S77-S86 unter https://doi.org/10.1055/a-1553-3565

\section{ZUSAMMENFASSUNG}

Ziel der Studie Routinedaten von Krankenkassen sind als Datenquelle mittlerweile gut etabliert. Hinsichtlich der Verallgemeinerbarkeit der Ergebnisse bei Analysen mit Daten einer Krankenkasse treten Fragen der Repräsentativität der Versichertenpopulation auf, insbesondere da nicht alle Studien auf soziodemografische Merkmale adjustieren. Diese Arbeit untersucht mittels deskriptiver Analyse, ob und inwieweit sich die Sozialstruktur der Versichertenpopulation der AOK Niedersachsen von der Sozialstruktur der Allgemeinbevölkerung und der sozialversicherungspflichtig Beschäftigten in Niedersachsen (NDS) und in der Bundesrepublik (BRD) unterscheiden. Methodik Die Datengrundlage bilden pseudonymisierte Daten der AOK NDS, die Beschäftigtenstatistik der Bundesagentur für Arbeit und der Bevölkerungsstand in NDS und der BRD. Die Versichertenpopulation wird an zwei Stichtagen 31.12.2012 und 31.12.2017 hinsichtlich der Geschlechter-und Altersstruktur mit der Bevölkerung in NDS und der BRD verglichen. Anschließend werden die Daten der sozialversicherungspflichtig Beschäftigten in der AOK NDS und aus der Beschäftigtenstatistik der Bundesagentur für Arbeit gegenübergestellt, um Ausbildungsabschlüsse, Komplexität der ausgeübten Tätigkeit und elf Berufsbereiche zu vergleichen.

Ergebnisse Die Geschlechterstruktur unterscheidet sich nicht zwischen den drei Vergleichspopulationen. Verglichen mit der Bevölkerung in NDS und der BRD ist der Anteil der unter 30-Jährigen in der AOK NDS überdurchschnittlich, der Anteil der Personen zwischen 50 und 76 Jahren etwas unterdurchschnittlich. Sozialversicherungspflichtig Beschäftigte mit Hochschulabschluss und in Tätigkeiten mit höherer Komplexität sind in der AOK NDS unterrepräsentiert. Die Verteilung der sozialversicherungspflichtig Beschäftigten auf elf Berufsbereiche unterscheidet sich ebenfalls.

Schlussfolgerung Die Studie zeigt, dass soziodemographische und sozio-ökonomische Merkmale in Studien mit Krank- 
enkassendaten wann immer möglich berücksichtigt werden sollten. In Zukunft wird das Informationssystem Versorgungsdaten krankenkassenübergreifende Analysen mit Sekundärdaten ermöglichen. Fragestellungen der gesundheitlichen Ungleichheit können damit jedoch aufgrund des Fehlens von sozio-ökonomischen Merkmalen nicht beantwortet werden. Auch die Identifikation von vulnerablen Gruppen, die gezielt geeigneten Maßnahmen zugeführt werden könnten, ist ohne Berücksichtigung von sozio-ökonomischen Merkmalen erschwert.

\section{ABSTRACT}

Aim of the work Routine data from statutory health insurance funds are now a well-established source of data for scientific research. With regard to the generalizability of findings based on data from one health insurance fund, questions arise regarding the representativeness of the insured population, especially since not all studies adjust for socio-demographic characteristics. Our study examines whether and to what extent socio-demographic and occupational characteristics of the population insured with the AOK Lower Saxony differ from the total and working population of Lower Saxony and the Federal Republic of Germany.

Methods The analyses are based on pseudonymised data from the AOK, the employment statistics of the Federal Em- ployment Agency (FEA) and population statistics. The insured population was compared with the population of Lower Saxony and Germany at two cut-off dates (31.12.2012 and 31.12.2017) with respect to the distributions of age and gender. Subsequently, data of employed insured persons were compared with FEA-data in order to compare educational level, complexity of the work and occupational areas.

Results The gender structure did not differ between the three populations. The proportion of insured women and men below 30 years of age was above the corresponding figures of Lower Saxony and Germany. Employed individuals holding a university degree or jobs with higher complexity were under-represented in the AOK. The distribution across eleven occupational areas in the AOK also differed from the reference populations. Conclusions The study shows that socio-demographic and socio-economic characteristics should be considered whenever possible in studies using statutory health insurance data. In future, the new database “Information System Health Care Data" will enable analyses across all statutory health insurance providers. However, research questions of health inequalities cannot be answered with this approach due to the lack of socio-economic characteristics in these data. Identifying vulnerable groups that could be targeted for appropriate interventions is also difficult without taking socio-economic characteristics into account.

\section{Einleitung}

Routinedaten von Krankenkassen sind in der Versorgungsforschung, in der Epidemiologie und in der Medizinischen Soziologie als Datenquelle mittlerweile gut etabliert. In den meisten Fällen basieren die Analysen auf Sekundärdaten einer einzelnen Krankenkasse [1]. Hinsichtlich der Verallgemeinerbarkeit der Ergebnisse treten jedoch immer wieder Fragen der Repräsentativität auf. Diese Arbeit untersucht, ob und inwieweit sich die soziodemografischen und sozio-ökonomische Merkmale der Versichertenpopulation der Allgemeinen Ortskrankenkasse Niedersachsen (AOKN) von der deutschen Bevölkerung unterscheiden.

Die Sozialstruktur der Versichertenpopulationen einzelner gesetzlicher Krankenkassen ist in Deutschland historisch entstanden. Trotz der Öffnung im Jahr 1996 muss davon ausgegangen werden, dass es zwischen Versichertenpopulationen von Kassen und der Gesamtbevölkerung der Bundesrepublik deutliche Unterschiede gibt [2]. Diese Frage wurde bisher nur auf Basis von Surveydaten und als Vergleich zwischen Krankenkassen behandelt, jedoch nicht in Relation zur Allgemeinbevölkerung. So haben Hoffmann und Icks die Sozialstruktur und Prävalenzen ausgewählter Erkrankungen bei Versicherten unterschiedlicher gesetzlicher Krankenkassen und privat Versicherter auf Basis von Befragungsdaten des Bertelsmann Gesundheitsmonitors [3] sowie der bundesweiten telefonischen Gesundheitsbefragung vom RKI GSTel [4] untersucht. Später nahmen Ramos und Hoffmann ähnliche Vergleiche auf Basis des Sozio-Ökonomischen Panels vor [5]. In all diesen Studien weisen AOK-Versicherte im Vergleich zu den Versichertenpopulationen anderer gesetzlicher oder der privaten Krankenversicherung einen geringeren Anteil von Personen mit Abitur auf. Beim Vergleich der Prävalenzen zeigten sich für AOK-Versicherte auch nach Kontrolle für mehrere soziodemographische Merkmale und Komorbiditäten erhöhte Werte für Diabetes ( $O R=1,73$ im Vergleich zu privat Versicherten) und Herzinsuffizienz $(O R=1,56)$ [3]. Unklar bleibt jedoch, in welchem Ausmaß Versichertenpopulationen hinsichtlich ihrer Sozialstruktur von der Allgemeinbevölkerung abweichen und wie sich die Besonderheiten eines Surveydesigns auf die Zusammensetzung der Stichprobe auswirkten.

Uns sind keine weiteren Studien bekannt, in denen Vergleiche zwischen Versichertenpopulationen einer Krankenkasse mit der Allgemeinbevölkerung durchgeführt wurden. Unsere eigene Publikation aus dem Jahr 2013 [6] hatte die Sozialstruktur der Allgemeinen Ortskrankenkasse Niedersachsen (AOKN) anhand der bis 30. November 2011 geltenden Kodierung mit zwei Schulabschlüssen und dreistelligen Berufscodes mit der niedersächsischen und deutschen Allgemeinbevölkerung verglichen. Die Berufscodierungen und Informationen zu Berufsbildungs-und Schulabschlüssen, die einen solchen Vergleich der Sozialstruktur ermöglichen, können einem sogenannten Tätigkeitsschlüssel entnommen werden. Der Tätigkeitsschlüssel ist Bestandteil der Datenmenge, die von Arbeitgebern im Rahmen des Meldeverfahrens zur Sozialversicherung übermittelt wird (mehr unter „Methoden“). Zum 1. Januar 2011 wurde der Tätigkeitsschlüssel erneuert [7] und besteht seitdem aus neun statt aus fünf Ziffern. Auf der Grundlage dieser aktuellen Klassifikation sind detailliertere Vergleiche möglich, die mit dieser Ar- 
beit vorgestellt werden. So können nach der aktuell geltenden Kodierung Berufsbildungsabschlüsse in sieben Kategorien ausgewertet werden (vorher drei), bei Berufsbezeichnungen kann das Anforderungsniveau bzw. die Komplexität der ausgeübten Tätigkeit wie auch die Zugehörigkeit des einzelnen Berufs zu einem von elf Berufsbereichen für eine Analyse herangezogen werden (vorher fünf Berufsgruppen).

Die vorliegende Analyse gliedert sich in zwei Teile: [1] Vergleich der Versichertenpopulation der AOKN mit der Bevölkerung Niedersachsens und der Bundesrepublik Deutschland (Fragen 1 und 2) und [2] Analyse der Subpopulation der sozialversicherungspflichtig Beschäftigten bei der AOKN mit der Subpopulation der sozialversicherungspflichtig Beschäftigten in Niedersachsen und in der Bundesrepublik (Fragen 3, 4 und 5). Folgende Fragestellungen liegen der Analyse zugrunde:

1. Unterscheiden sich die Geschlechter-und Altersstruktur in der Versichertenpopulation der AOKN von der Bevölkerungsstruktur Niedersachsens und der Bundesrepublik Deutschland?

2. Unterscheiden sich die Anteile sozialversicherungspflichtig Beschäftigter in der AOKN im Vergleich zu sozialversicherungspflichtig Beschäftigten in NDS und der BRD?

3. Unterscheiden sich die Ausbildungsabschlüsse der sozialversicherungspflichtig Beschäftigten zwischen den drei Populationen?

4. Unterscheidet sich die Aufteilung der sozialversicherungspflichtig Beschäftigten nach der Komplexität der ausgeübten Tätigkeit zwischen den drei Populationen?

5. Welche Branchen bzw. Berufsbereiche sind wie häufig unter den sozialversicherungspflichtig Beschäftigten in den drei Populationen vertreten?

Um eine Entwicklung über die Zeit in allen drei Populationen vergleichend darzustellen, wurden im Rahmen der Analyse zwei Stichtage verwendet: 31.12.2012 und 31.12.2017. Das Jahr 2017 war zum Zeitpunkt der Analysen das aktuellste verfügbare Jahr. Das Jahr 2012 wurde so gewählt, dass der Vergleich über die Zeit möglichst weit auseinander liegende Zeitpunkte enthielt, die Daten jedoch auf Basis der gleichen Kodierung dokumentiert worden waren.

\section{Methoden}

Bei dieser Studie handelt es sich um eine deskriptive vergleichende Analyse der Sozialstruktur der AOKN, der Bevölkerung Niedersachsens und der Bundesrepublik. Die Datengrundlage der folgenden Analysen bilden folgende Datenquellen: 1) pseudonymisierte Daten der AOKN, 2) der fortgeschriebene Bevölkerungsstand auf Basis der Ergebnisse des Zensus aus dem Jahr 2011 [8] und 3) die Beschäftigtenstatistik der Bundesagentur für Arbeit [9]. Dabei handelt es sich bei der Beschäftigtenstatistik der Bundesagentur für Arbeit um aggregierte Tabellen, die die Anzahl der sozialversicherungspflichtig Beschäftigten in Niedersachsen bzw. Deutschland stratifiziert nach unterschiedlichen Merkmalen zeigen. In dieser Studie wurden entsprechende Tabellen mit Stratifizierung nach Geschlecht, Altersgruppen, Berufsbildungsabschluss, Berufsbereich und Tätigkeitskomplexität verwendet. Aus den Datenquellen 1 und 2 wurden die Angaben zum Geschlecht und Alter verwendet (Fragen 1 und 2). Die Analyse der Merkmale der sozialversicherungs- pflichtig Beschäftigten in Fragen 3, 4 und 5 (Berufsbildungsabschluss, Komplexität der ausgeübten Tätigkeit sowie Berufsbereich) basierte auf den Datenquellen 1 und 3. Im Folgenden wird das Vorgehen und die einzelnen Merkmale genauer beschrieben.

Aus den AOKN-Daten wurden nur Personen berücksichtigt, die am Stichtag 31.12.2012 bzw. 31.12.2017 versichert waren. Für die Analyse der sozialversicherungspflichtig Beschäftigten (SVPB) wurden in den AOKN-Daten nur Personen berücksichtigt, die am entsprechenden Stichtag als SVPB versichert waren.

Zur Beantwortung der Fragestellungen 3, 4 und 5 wurden die Berufscodierungen und Informationen zu Berufsbildungsabschlüssen dem Tätigkeitsschlüssel entnommen. Nach der Datenerfassungs-und-übermittlungsverordnung (DEÜV) sind Arbeitgeber im Rahmen des Meldeverfahrens zur Sozialversicherung verpflichtet, verschiedene Informationen zu ihren Beschäftigten an die Einzugsstelle zu übermitteln, inklusive des Tätigkeitsschlüssels [10]. Laut $\S 28$ i des SGB IV ist die für den Arbeitnehmer zuständige Krankenkasse eine solche Einzugsstelle und erhält dementsprechend diese Daten, die sie aufbereitet und an andere Träger der Sozialversicherung weiterleitet ( $§ 33$ der DEÜV). Die Beschäftigtenstatistik der Bundesagentur für Arbeit basiert ebenfalls auf diesen Daten ( $\$ 36$ [3] der DEÜV) [11], d. h. die Daten für sozialversicherungspflichtig Beschäftigte in allen drei Subpopulationen folgten dem gleichen Datenerfassungsprozess.

Der zum 1. Januar 2011 aktualisierte Tätigkeitsschlüssel [7] besteht aus neun Ziffern. Die ersten fünf Stellen definieren den Berufscode, in der siebten Stelle ist der Ausbildungsabschluss vermerkt [12]. Die fünfstelligen Berufscodes wurden in zwei Auswertungen verwendet: Zum einen wurde die letzte der fünf Stellen verwendet, um die Komplexität der ausgeübten Tätigkeit abzubilden (Frage 4). Die Berufsbezeichnung enthält entsprechende Ergänzungen: [1] Helfer-und Anlerntätigkeiten, [2] fachlich ausgerichtete Tätigkeit, [3] komplexe Spezialistentätigkeiten und Aufsichtskräfte und [4] hoch komplexe Tätigkeiten und Führungskräfte [13].

Die zweite Auswertung des fünfstelligen Berufscodes (Frage 5) bezieht sich auf elf Berufsbereiche die aus den ersten beiden Stellen des Berufscodes extrahiert wurden und eine übergeordnete Ebene von Branchenbezeichnungen darstellen (z. B. Handel und Tourismus oder Büro, Finanzen und Verwaltung). Die Klassifikation in elf Berufsbereiche ist an die Klassifikation im Leitfaden der Bundesagentur für Arbeit angelehnt [13]. Die Unterschiede betreffen lediglich den großen Berufsbereich 2: Rohstoffgewinnung, Produktion und Fertigung im Leitfaden der Bundesagentur für Arbeit, der in der vorliegenden Arbeit in drei Untergruppen 2: Rohstoffgewinnung und-verarbeitung, 3: Maschinen-und Fahrzeugtechnik; Energie und Elektrik; Konstruktion und 4: Textil und Lebensmittel aufgeteilt wurde.

Die Analyse der soziodemografischen und sozio-ökonomischen Merkmale der drei Populationen erfolgt deskriptiv und meist nach Geschlecht stratifiziert.

\section{Ergebnisse Teil 1}

- Tab. 1 zeigt, dass sich die Geschlechteranteile zwischen den drei Populationen nur gering unterscheiden. Der Anteil der Frauen unter den AOKN-Versicherten lag im Jahr 2012 mit 52,1 \% marginal höher als in NDS (51,5\%) und der BRD (51,6\%). Diese Unterschiede ver- 
- Tab. 1 Zusammensetzung der Versicherten der AOK Niedersachsen und der Bevölkerung Niedersachsens und der Bundesrepublik nach Geschlecht ab 18 Jahre (Stichtag 31.12.2012 und 31.12.2017).

\begin{tabular}{|c|c|c|c|c|c|c|c|c|c|}
\hline & \multicolumn{3}{|c|}{ AOK Niedersachsen ${ }^{1)}$} & \multicolumn{3}{|c|}{ Bevölkerung Niedersachsen²) } & \multicolumn{3}{|c|}{ Bevölkerung Deutschland²) } \\
\hline & Männlich & Weiblich & Gesamt & Männlich & Weiblich & Gesamt & Männlich & Weiblich & Gesamt \\
\hline \multirow[t]{2}{*}{2012} & $47,9 \%$ & $52,1 \%$ & $100,0 \%$ & $48,5 \%$ & $51,5 \%$ & $100,0 \%$ & $48,4 \%$ & $51,6 \%$ & $100,0 \%$ \\
\hline & 968609 & 1053126 & 2021735 & 3128770 & 3319907 & 6448677 & 32664323 & 34768536 & 67432859 \\
\hline \multirow[t]{2}{*}{2017} & $48,8 \%$ & $51,2 \%$ & $100,0 \%$ & $49,0 \%$ & $51,0 \%$ & $100,0 \%$ & $48,9 \%$ & $51,1 \%$ & $1000 \%$ \\
\hline & 1083927 & 1137874 & 2221801 & 3247984 & 3385614 & 6633598 & 33875273 & 35378932 & 69254205 \\
\hline
\end{tabular}

1) Datenquelle: pseudonymisierte Daten der AOK Niedersachsen. 2) Datenquelle: der fortgeschriebene Bevölkerungsstand auf Basis der Ergebnisse des Zensus aus dem Jahr 2011.

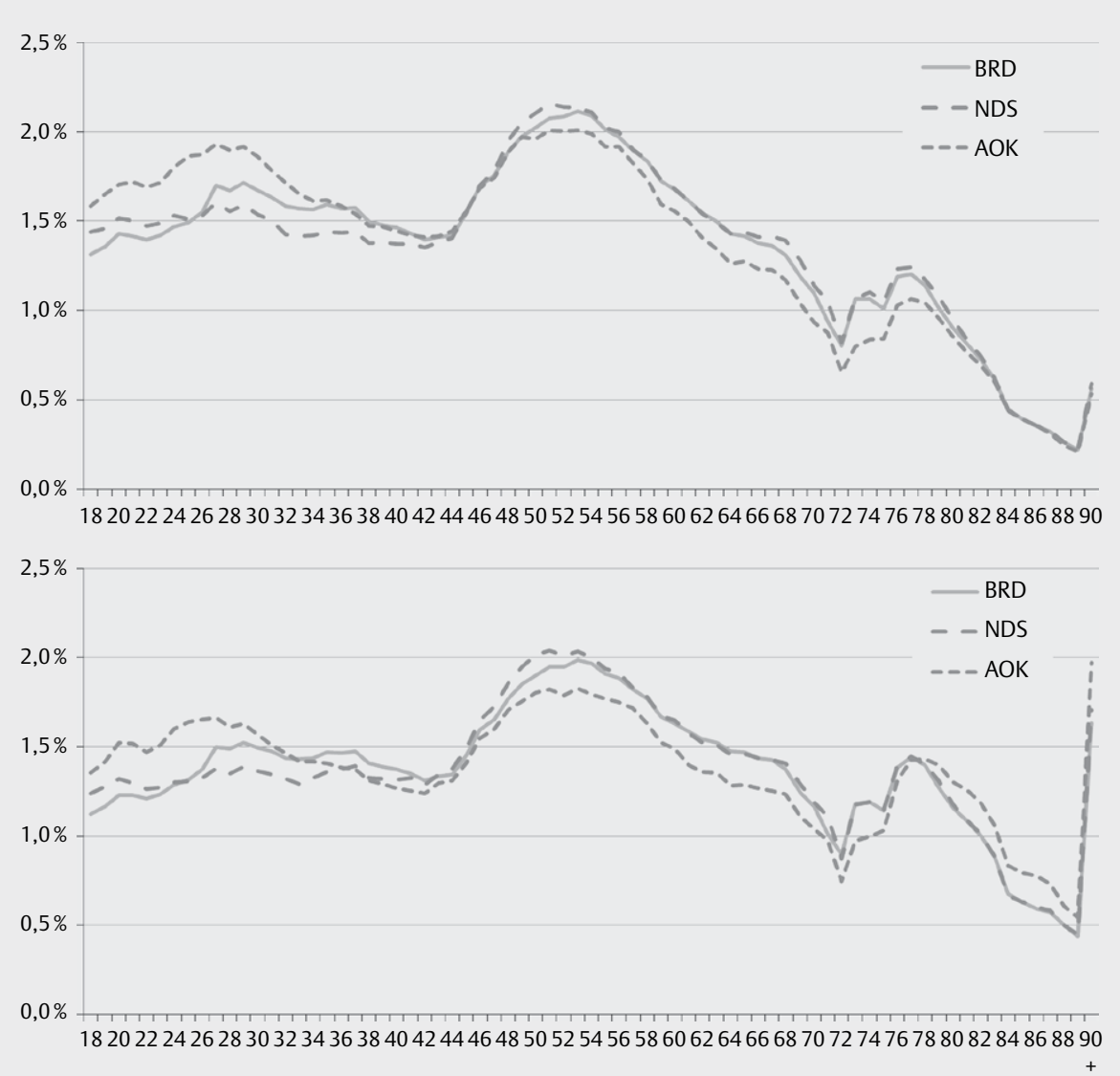

Abb. 1 Altersstruktur der Versicherten der AOK Niedersachsen und der Bevölkerung Niedersachsens und der Bundesrepublik (Stichtag 31.12.2017) ab dem Alter von 18 Jahren für Männer (oben) und Frauen (unten).

ringerten sich über die Zeit noch und fielen auf 0,1 bzw. 0,2 Prozentpunkte im Jahr 2017.

Der zweite Blick gilt der Altersstruktur der drei untersuchten Populationen. In der $>$ Abb. 1 sind die Anteile der jeweiligen Altersstufen im Jahre 2017 getrennt für Männer (oben) und Frauen (unten) dargestellt. In der AOKN war der Anteil junger Personen unter 30 Jahren höher als in der Bevölkerung in NDS und der BRD. Dieser Unterschied ist bei Männern etwas stärker ausgeprägt als bei Frauen. In der Altersgruppe zwischen 50 und 76 Jahren war der Anteil an der Gesamtversichertenpopulation der AOKN hingegen etwas geringer als in der niedersächsischen und gesamtdeutschen Bevölkerung. Der Anteil der älteren Frauen (80 jahre und älter) lag in der AOKN mit 11,1 \% höher als in NDS (9,3\%) oder in der BRD $(9,1 \%)$. Bei Männern ergaben sich keine nennenswerten Unterschiede zwischen den drei Populationen ab dem Alter von 80 Jahren.

Mit Blick auf den Anteil der sozialversicherungspflichtig Beschäftigten ist in allen drei Populationen ein Anstieg über die Zeit erkennbar ( $\triangleright$ Tab. 2). Zu beiden Stichtagen waren die Anteile der SVPB unter Frauen der AOKN niedriger als in der niedersächsischen bzw. bundesweiten Bevölkerung. Diese Differenzen haben sich über die Zeit verringert: So lag der Unterschied zu NDS im Jahr 2012 bei 5,2 Prozentpunkten und zur BRD bei 6,4 Prozentpunkten und verringerte sich im Jahr 2017 auf 2,2 und 3,3 Prozentpunkte entsprechend. Somit verzeichnete die AOKN einen stärkeren Anstieg im 


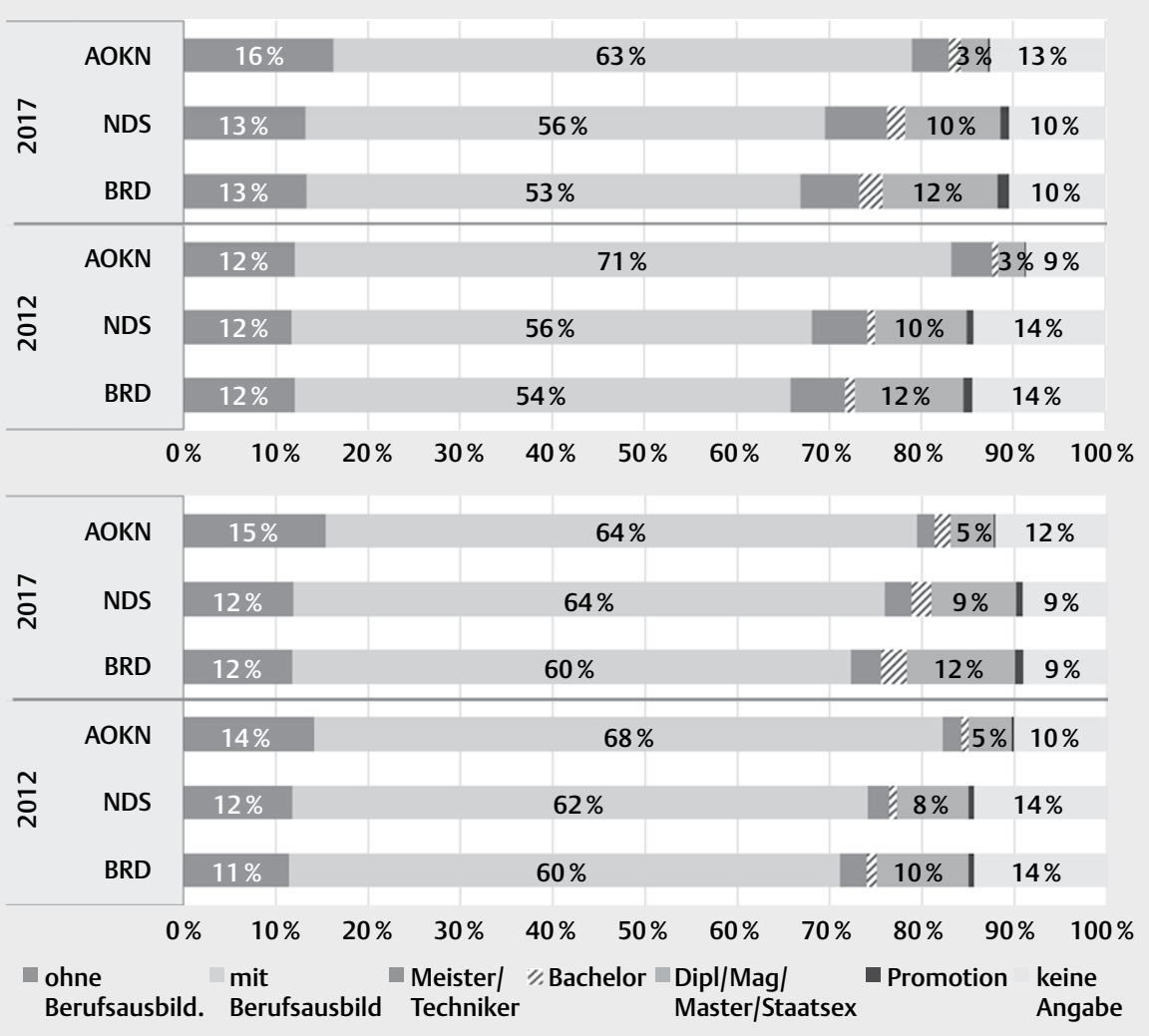

- Abb. 2 Sozialversicherungspflichtig Beschäftigte bei der AOK Niedersachsen, in Niedersachsen und bundesweit nach dem höchsten berufsbildenden Abschluss in 2012 und 2017 für Männer (oben) und Frauen (unten).

Anteil sozialversicherungspflichtig beschäftigter Frauen über die Zeit als die Vergleichspopulationen. Für Männer sind die Unterschiede insgesamt deutlich geringer. Detailliertere Vergleiche nach Altersgruppen sind im Online-Supplement für die beiden Stichtage dargestellt ( $>$ Abb. S1 und $>\mathbf{S 2}$ im Online-Supplement). Diese Zusatzanalysen zeigen, dass die Angleichung der Anteile von sozialversicherungspflichtig beschäftigten Frauen zwischen der AOKN und den beiden Vergleichspopulationen in allen Altersgruppen stattgefunden hat.

\section{Ergebnisse Teil 2}

In folgenden Abschnitt werden vergleichende Analysen für die Subpopulationen der sozialversicherungspflichtig Beschäftigten (SVPB) vorgestellt. Hinsichtlich der Qualifikation der SVPB finden sich für das Jahr 2017 bei den Versicherten der AOKN im Vergleich zu sozialversicherungspflichtig Beschäftigten in NDS oder der BRD etwas höhere Anteile von Personen ohne abgeschlossene Berufsausbildung ( $\triangleright$ Abb. 2). Dies gilt auch im Jahr 2012 für Frauen. Über die Zeit ist der Anteil von Personen mit Hochschulabschluss in allen drei Subpopulationen gestiegen, dabei war der Anstieg bundesweit jedoch deutlicher $(+24,0 \%)$ als in Niedersachsen ( + 22,2\%) oder bei der AOKN ( + 20,6\%). Unter den AOKN-versicherten SVPB fällt der Anteil der Personen mit Hochschulabschluss im Jahr 2017 mit 4,7\% deutlich niedriger aus als in der BRD $(15,9 \%)$ oder in NDS $(12,8 \%)$.

Die neue Klassifikation der Berufe KIdB2010 ermöglicht eine Analyse der Subpopulation der sozialversicherungspflichtig Beschäftigten nach der Komplexität der ausgeübten Tätigkeit. Wie aus der - Abb. 3 ersichtlich, ist der Anteil der Personen mit komplexeren Berufen unter den sozialversicherungspflichtig Beschäftigten in der AOKN geringer als in der entsprechenden niedersächsischen und gesamtdeutschen Subpopulation. Dabei ist der Anteil der beiden Berufsgruppen mit höherer Tätigkeitskomplexität unter den AOKNVersicherten über die Zeit etwas gestiegen (von 9,7\% auf $11 \%$ ). Dagegen ist dieser Anteil in NDS (22\%) und in der BRD (ca. 25\%) eher stabil geblieben.

Die Berufscodes geben außerdem Aufschluss über die Branchen bzw. Berufsbereiche. In Anlehnung an die Bundesagentur für Arbeit [13] wurde in dieser Analyse eine Differenzierung in elf Berufsbereiche vorgenommen.

Die Anteile je Berufsbereich ähneln sich zwischen den bundesweiten und niedersächsischen Subpopulationen. Beim Vergleich der AOKN-Versicherten zu den beiden Vergleichssubpopulationen fallen einige Unterschiede auf: In den Berufsbereichen, die meist Tätigkeiten im Büro enthalten, wie bspw. [9] Büro, Finanzen und Verwaltung oder [1] Geisteswissenschaft und Werbung sind die Anteile unter den Versicherten der AOKN deutlich geringer als in der bundesweiten und der landesweiten Subpopulation der sozial- 


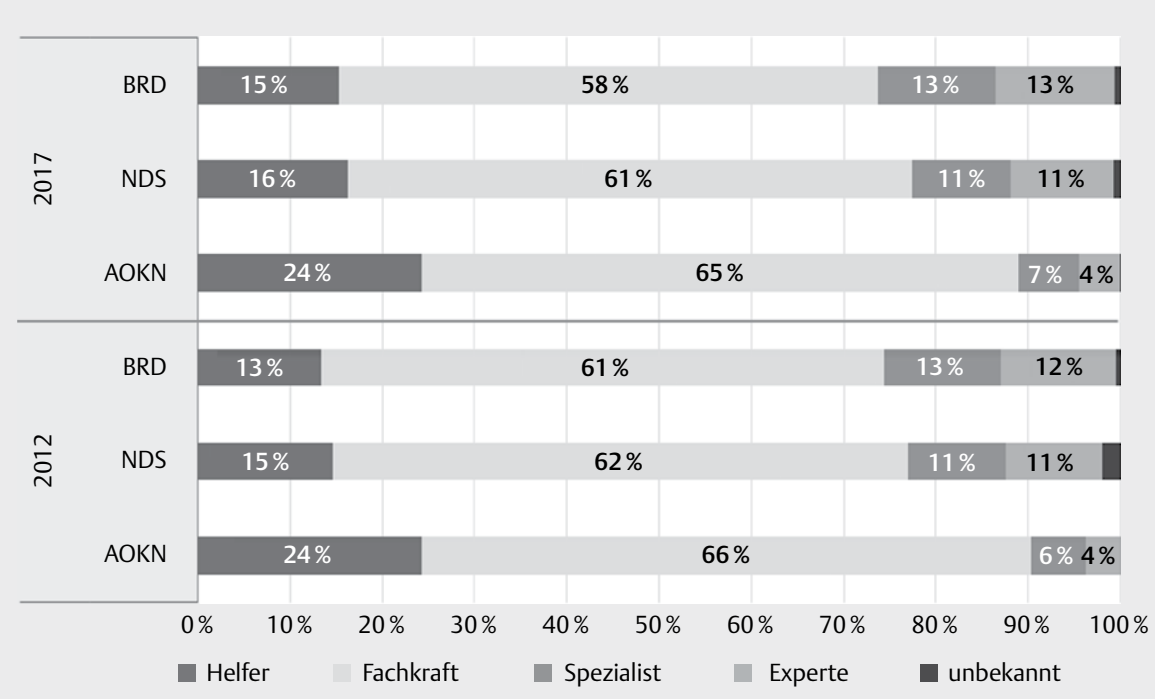

- Abb. 3 Sozialversicherungspflichtig Beschäftigte unter den Versicherten der AOK Niedersachsen, in Niedersachsen und der Bundesrepublik Deutschland nach der Tätigkeitskomplexität des Berufscodes.

versicherungspflichtig Beschäftigten. Dagegen sind die Berufsbereiche mit vorwiegend körperlichen Tätigkeiten wie [5] Bau und Versorgungstechnik oder [7] Verkehr, Sicherheit, Reinigung in der AOKN überrepräsentiert im Vergleich zu NDS oder der BRD. Diese Vergleiche gelten für Männer und Frauen gleichermaßen. Lediglich im Berufsbereich [8] Handel und Tourismus unterscheidet sich die Richtung der Vergleiche: So sind unterdurchschnittlich viele bei der AOKN versicherte Männer und überdurchschnittlich viele bei der AOKN versicherte Frauen in diesem Berufsbereich tätig, verglichen mit sozialversicherungspflichtig Beschäftigten in NDS und der BRD.

\section{Diskussion}

Als erste gesetzliche Krankenkasse in Deutschland ermöglicht die AOK Niedersachsen mit dieser Analyse den Blick auf die soziodemografische Struktur ihrer Versicherten, insbesondere der Subpopulation der sozialversicherungspflichtig Beschäftigten, und erlaubt somit den Vergleich zur Gesamtbevölkerung in NDS und der BRD. Über die Geschlechter- und Altersstruktur hinaus wurden vertiefende Vergleiche für sozialversicherungspflichtig beschäftigte Personen hinsichtlich der Ausbildungsabschlüsse, der Komplexität der von ihnen ausgeübten Tätigkeiten und der Berufsbereiche bzw. Branchen durchgeführt.

Im ersten Teil der Studie wurden die Geschlechter- und Altersstruktur der Versichertenpopulation der AOKN mit der Bevölkerung in Niedersachsen und bundesweit verglichen (Fragen 1 und 2). Hinsichtlich der Geschlechterverhältnisse unterscheidet sich die AOKN im Jahr 2017 nicht von der Bevölkerung in NDS oder der BRD. Die Altersstruktur zeigt einen höheren Anteil jüngerer Erwachsener unter 30 Jahren in der AOKN wie auch einen etwas geringeren Anteil der Personen zwischen 50 und 76 Jahren als dies der Struktur der niedersächsischen oder bundesweiten Bevölkerung entspricht. In anderen Altersstufen bestehen nur sehr geringe Diskrepanzen zwischen den drei Vergleichspopulationen.
Diese Ergebnisse unterscheiden sich von Analysen auf Basis des Gesundheitsmonitors [2, 3], nach denen die AOK-Versicherten einen höheren Anteil von älteren Personen aufweisen. Die Unterschiede zwischen unseren und den Analysen auf der Grundlage von Surveydaten erarbeiteten Befunde könnten durch selektiven Nonresponse oder durch Besonderheiten der Stichprobenziehung erklärt werden. Außerdem stammen die genannten Studien aus einer anderen Zeit, und die Sozialstruktur von Krankenversicherungen muss auch als über die Zeit veränderlich angesehen werden. Seit 2010 sind netto insgesamt ca. 420 Tausend Versicherte zur AOK Niedersachsen gewechselt. Kassenwechsler sind im Allgemeinen deutlich jünger als der gesamtdeutsche Altersdurchschnitt [14], so dass sich die Altersstruktur der AOK über die Jahre deutlich gewandelt hat.

Im Teil 2 wurden die Subpopulationen der sozialversicherungspflichtig Beschäftigten verglichen (Fragen 3, 4 und 5). Hier zeigten sich größere Unterschiede zwischen den Subpopulationen: So ist der Anteil der Personen mit Hochschulabschluss unter den Beschäftigten in der AOKN deutlich niedriger als in NDS oder in der BRD. Damit verbunden ist auch der Anteil der Personen in Berufen mit höherer Tätigkeitskomplexität, der in der AOKN ebenfalls kleiner ausfällt aus in den Vergleichspopulationen. Ein Grund liegt möglicherweise in den überdurchschnittlich hohen Anteilen der Beschäftigten in Berufsbereichen wie bspw. [7] Verkehr, Sicherheit und Reinigung oder [5] Textil und Lebensmittel, wo Berufe mit Hochschulabschluss seltener vertreten sind.

Diese Perspektive auf die Berufsbereiche oder Branchen wird in den bisherigen Vergleichsstudien zwischen Versicherten unterschiedlicher gesetzlicher und privater Krankenversicherungen nicht berücksichtigt. In der Vergleichsstudie auf Basis von Befragungsdaten des Gesundheitsmonitors [3] wurde bei der Berechnung von Prävalenzen für mehrere chronische Erkrankungen für Schulabschluss, Komorbiditäten und weitere gesundheitsbezogene Variablen wie Rauchen kontrolliert. Verglichen mit Privatversicherten zeigten sich für AOK-Versicherte erhöhte Prävalenzen von Diabe- 


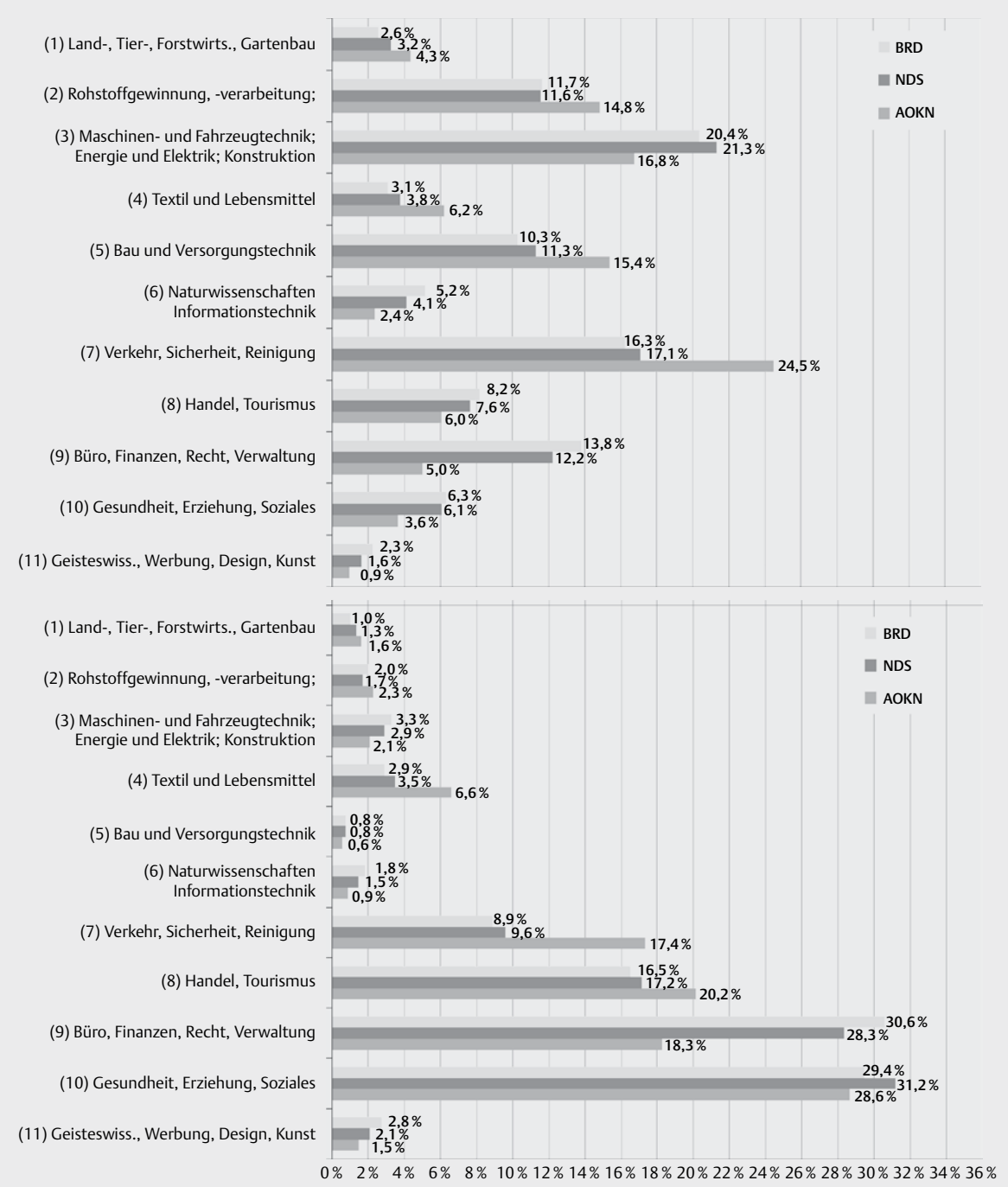

- Abb. 4 Sozialversicherungspflichtig Beschäftigte unter den Versicherten der AOK Niedersachsen, in Niedersachsen und bundesweit nach elf Berufsbereichen anteilig innerhalb der jeweiligen Erwerbsbevölkerung am Stichtag 31.12.2017 für Männer (oben) und Frauen (unten).

tes und Herzinsuffizienz. Die Vermutung liegt nahe, dass andere Faktoren die Erkrankungsrisiken beeinflussen, die möglicherweise mit beruflichen Belastungen zusammenhängen. Nach dem Anforderungs-Kontroll-Modell nach Karasek und Theorell [15] kommt es zu beruflichen Stressbelastungen, wenn im Beruf ein Ungleichgewicht zwischen hohen Anforderungen und geringen Kontrollmöglichkeiten bei der Ausübung der Tätigkeit entsteht. Diese chronische Stressbelastung versetzt den Körper in einen dauerhaften Aktivierungszustand, der zu Erschöpfung und in der Folge zu erhöhten Erkrankungsrisiken führt. Eine solche Stressbelastung ist häufiger bei Berufen in den Bereichen Sicherheit, Reinigung oder Lebensmittel zu vermuten, insbesondere wenn es sich um weniger komplexe ausführende Tätigkeiten handelt, wie es bei AOKN-Versicherten überdurchschnittlich häufig der Fall ist ( $\mathbf{A} \mathbf{A b} \mathbf{b}$. 3). Der Link von chronischem Stress zur Entstehung von Diabetes Typ 2 wird dabei oft über die erhöhte Entzündungsreaktion des Immun- systems vermutet, wenn das Individuum über längere Zeit Anspannung oder Stress verspürt $[16,17]$.

In einer weiteren Analyse des Sozio-Ökonomischen Panels konnten Ramos und Hoffmann zeigen, dass die Prävalenzen von Gelenkerkrankungen und chronischen Rückenbeschwerden bei den AOK-Versicherten erhöht waren [5]. Auch hier wurde nach Alter und Geschlecht standardisiert, jedoch nicht für sozioökonomische Merkmale oder Branche adjustiert. Dabei fällt in der hier vorliegenden Analyse auf, dass in den körperlich anspruchsvollen Berufen aus den Berufsbereichen [2] Rohstoffgewinnung und-verarbeitung wie auch [5] Bau und Versorgungstechnik die Anteile der AOKNVersicherten höher sind als unter den sozialversicherungspflichtig Beschäftigten in der Allgemeinbevölkerung, was die Unterschiede in der Prävalenz von Rückenbeschwerden erklären könnte.

Vor dem Hintergrund der hier vorgestellten Ergebnisse ist es daher zentral, für die soziodemographischen Unterschiede zwischen der Krankenversicherungspopulation und der Allgemeinbev- 
- Tab. 2 Anteil der sozialversicherungspflichtig Beschäftigten unter der Versicherten der AOK Niedersachsen, in der Bevölkerung Niedersachsens und der Bundesrepublik Deutschland nach Geschlecht (Stichtag 31.12. des jeweiligen Jahres).

\begin{tabular}{|c|l|l|l|l|l|l|l|l|l|}
\hline & \multicolumn{3}{|c|}{ AOK Niedersachsen1) } & \multicolumn{2}{c|}{ Bevölkerung Niedersachsen ${ }^{2)}$} & \multicolumn{3}{c|}{ Bevölkerung Deutschland2) } \\
\cline { 2 - 10 } & Männlich & Weiblich & Gesamt & Männlich & Weiblich & Gesamt & Männlich & Weiblich & Gesamt \\
\hline \multirow{2}{*}{2012} & $48,2 \%$ & $32,7 \%$ & $100,0 \%$ & $48,9 \%$ & $37,9 \%$ & $43,2 \%$ & $48,2 \%$ & $39,1 \%$ & $43,5 \%$ \\
\cline { 2 - 10 } & 467217 & 344659 & 811876 & 1531085 & 1257433 & 2788518 & 15756304 & 13602750 & 29359054 \\
\hline \multirow{2}{*}{2017} & $51,3 \%$ & $39,3 \%$ & $45,1 \%$ & $51.3 \%$ & $41,5 \%$ & $46,3 \%$ & $51,1 \%$ & $42,6 \%$ & $46,8 \%$ \\
\cline { 2 - 9 } & 555892 & 446793 & 1002685 & 1665902 & 1405605 & 3071507 & 17322312 & 15068754 & 32391066 \\
\hline
\end{tabular}

1) Datenquelle: pseudonymisierte Daten der AOK Niedersachsen; 2) Datenquelle: die Beschäftigtenstatistik der Bundesagentur für Arbeit (Zähler) und der fortgeschriebene Bevölkerungsstand auf Basis der Ergebnisse des Zensus aus dem Jahr 2011 (Nenner).

ölkerung zu adjustieren, insbesondere wenn Vergleiche zur gesamtdeutschen Bevölkerung angestrebt werden. Steht die Gesundheit von Personen im erwerbsfähigen Alter im Fokus der Studie, sollten, wann immer möglich, auch Unterschiede in den sozioökonomischen Merkmalen berücksichtigt werden.

In der eigenen Arbeitsgruppe wurde die Komplexität der ausgeübten Tätigkeit als Stratifizierungsmerkmal bereits in mehreren Analysen verwendet. So konnten Safieddine und Mitautoren zeigen, dass die Diabetesprävalenz für Männer in Berufen mit höchster Komplexität („Experten“) nach Kontrolle für das Alter, Einkommen und Bildung bei OR =0,93 lag (95\%-Konfidenzintervall: 0,880,99; $\mathrm{p}<0,05)$ im Vergleich zu Männern mit Hilfstätigkeiten als Referenzkategorie [18] (Frauen entsprechend: OR=0,78, 95\%-KI: 0,72-0,95; $\mathrm{p}<0,001)$. Für Multimorbidität zeigten Tetzlaff und Mitautoren, dass die Prävalenzen ebenfalls signifikant niedriger lagen für Personen in Berufen mit einer höheren Tätigkeitskomplexität, verglichen mit den Berufen auf dem niedrigsten Niveau der Komplexität [19]. In diesen Analysen wurde ebenfalls für das Alter, Einkommen und Bildung kontrolliert. In einer weiteren Studie wurde die Inanspruchnahme von antragspflichtiger Psychotherapie in Abhängigkeit vom Berufsbildungsabschluss, Einkommen und der Komplexität der ausgeübten Tätigkeit untersucht. Epping und Mitautoren fanden heraus, dass nach Kontrolle für Alter, Einkommen und Berufsbildungsabschluss Personen in Berufen mit höherer Komplexität der ausgeübten Tätigkeit eine zweifach erhöhte Chance zeigten, antragspflichtige Psychotherapie in Anspruch zu nehmen [20].

\section{Stärken und Limitationen}

Die hier verwendeten Versichertendaten der AOK enthalten detaillierte Informationen aus dem Versichertenverlauf wie Versichertenart und Tätigkeitsschlüssel, um Merkmale wie den Berufsbildungsabschluss, das Komplexitätsniveau der ausgeübten Tätigkeit und den Berufsbereich zu extrahieren.

Im Gegensatz zu anderen Vergleichsanalysen zwischen Krankenkassenpopulationen, die auf Befragungsdaten basieren, spielen Response-Bias, Erinnerungsbias oder Morbiditätsbias keine Rolle [21-24]. Die Vollständigkeit der Daten ist unabhängig von der Antwortbereitschaft oder der physischen wie psychischen Fähigkeit, an einer Befragung teilzunehmen oder sich richtig an eine Diagnose zu erinnern [25].

Des Weiteren wurde in dieser Studie der Vergleich der Versichertenstruktur einer Krankenkasse mit der Allgemeinbevölkerung vorgenommen. Die bisherigen Vergleichsstudien haben die Versi- chertenpopulationen einzelner gesetzlicher Krankenkassen und der privaten Krankenversicherungen einander gegenübergestellt und erlaubten somit keine Aussagen zur Repräsentativität für die Allgemeinbevölkerung.

Für das Geschlecht und das Geburtsjahr hängt die Genauigkeit der Angaben im Datenbestand der AOKN von Sachbearbeitern der einzelnen Geschäftsstellen der AOKN. Für die in den AOK-Daten enthaltenen Angaben zum Berufsbildungsabschluss, zur Komplexität der ausgeübten Tätigkeit wie auch zum Berufsbereich sind Personalsachbearbeiter in Organisationen oder Unternehmen verantwortlich, in denen die Versicherten tätig sind. Die zum Vergleich gezogenen Daten zu sozialversicherungspflichtig Beschäftigten in Niedersachsen und bundesweit entstammen der gleichen Datenbasis, die im Rahmen des Meldeverfahrens zur Sozialversicherung entsteht und an die Krankenkassen als Sozialversicherungsträger wie auch an die Bundesagentur für Arbeit entsprechend $\$ 36$ der DEÜV übermittelt wird $[10,11]$. Es ist vorstellbar, dass die Umstellung auf die neue Klassifikation der Berufe im Jahr 2011 nicht in allen Unternehmen fehlerfrei bis 2012 durchgeführt wurde. Es gibt keinen Grund zur Annahme, dass die damit einhergehenden Fehler in der Kodierung der Berufe in den drei zum Vergleich gezogenen Subpopulationen unterschiedlich häufig ausgeprägt sind.

\section{Ausblick und Schlussfolgerung}

Eine Veränderung hinsichtlich der für Routinedatenforschende verfügbaren Datenbasis könnte das sogenannte „Informationssystem Versorgungsdaten “ 1 mit sich bringen, das vom Deutschen Institut für Medizinische Dokumentation und Information (DIMDI) betreut wird²: Seit April 2015 besteht die Möglichkeit, Analysen mit dieser krankenkassenübergreifenden Datenbasis durchzuführen [26]. Dieser Datenbestand basiert noch bis zum Berichtsjahr 2018 auf den Informationen, die jährlich für die Umsetzung des Morbiditätsorientierten Risikostrukturausgleichs (Morbi-RSA) von den Krankenkassen an das Bundesversicherungsamt übermittelt wurden. Entsprechend den Vorgaben für den Morbi-RSA enthält dieser Datenbestand keine Inanspruchnahmedaten von Verstorbenen in ihrem Todesjahr.

\footnotetext{
1 früher auch als DaTraV-Datensatz unter den Routinedatenforschenden bekannt.

2 Seit Mai 2020 zusammengeführt mit dem Bundesinstitut für Arzneimittel und Medizinprodukte (BfArM) [23].
} 
Ab dem Berichtsjahr 2019 tritt die neue am 19. Juni 2020 verabschiedete Datentransparenzverordnung in Kraft, die das Übermitteln aller Krankenkassendaten an das Forschungsdatenzentrum (FDZ) [27] beim Bundesinstitut für Arzneimittel und Medizinprodukte für im §303e Absatz 2 SGB V aufgeführte Nutzungszwecke vorsieht [28]. Die im genannten FDZ gespeicherten Daten werden künftig auch Inanspruchnahmedaten für verstorbene Personen in ihrem Todesjahr enthalten. Das Feld Tätigkeitsschlüssel, aus dem die Berufsausbildung wie auch weitere hier ausgewertete berufliche Merkmale entnommen wurden, ist auch in der neuen Datentransparenzverordnung nicht vorgesehen [28]. Somit sind Stratifizierungen oder Adjustierungen nach soziodemografischen und sozioökonomischen Merkmalen bei Analysen mit dem „Informationssystem Versorgungsdaten“ nicht möglich. Auch die Identifikation von vulnerablen Gruppen erscheint dadurch erschwert.

Die Bereitstellung der Daten aus dem „Informationssystem Versorgungsdaten“ erfolgt nach $\S \S 303 a$ bis 303 f Sozialgesetzbuch V sowie der Datentransparenzverordnung $[10,28]$. Die Analyse dieser Daten ist erst nach der Bewilligung eines Antrags möglich, der an das FDZ gestellt werden muss. Daraufhin erstellen die Forschenden auf Basis von Testdatensätzen ein Auswertungsskript und erhalten nach einigen Monaten "standardisierte Datensätze in aggregierter und anonymisierter Form, ([28]S. 7), die von Forschenden auf Plausibilität und Vollständigkeit geprüft werden müssen.

Aufgrund des Zeitaufwands für den Zugang zu diesen Daten wäre anzunehmen, dass Forschende vorerst weiterhin mit einzelnen Krankenassen zusammenarbeiten werden. Außerdem stehen für Analysen der Zeiträume vor 2019 keine Inanspruchnahmedaten aus dem Todesjahr zur Verfügung. Somit werden vorerst weiterhin die meisten Studien auf Daten von einzelnen Krankenkassen basieren, die Kooperationen mit einzelnen Forschungseinrichtungen eingegangen sind. Bisher ist jedoch nicht bekannt, inwiefern sich die Versichertenpopulationen anderer gesetzlicher Krankenkassen von der Allgemeinbevölkerung unterscheiden.

\section{Interessenkonflikt}

Die Publikation entstand in einem Forschungsprojekt, das in Teilen durch die Forschungsförderung von der AOK Niedersachsen an SG finanziert wurde. JE's und JT's Stellen wurden in den letzten drei Jahren in Teilen durch diese Forschungsförderung finanziert.

\section{Literatur}

[1] Kreis K, Neubauer S, Klora M, Lange A, Zeidler J. Status and perspectives of claims data analyses in Germany-2014; A systematic review. Health Policy 2016; 120: 213-226

[2] Schnee M. Sozioökonomische Strukturen und Morbidität in den gesetzlichen Krankenkassen. In: Böcken J, Braun B, Amhof R, Hrsg. Gesundheitsmonitor 2008. Gesundheitsversorgung und Gestaltungsoptionen aus der Perspektive der Bevölkerung. Verlag Bertelsmann Stiftung; Gütersloh: 2008: 88-104

[3] Hoffmann F, Icks A. Unterschiede in der Versichertenstruktur von Krankenkassen und deren Auswirkungen für die Versorgungsforschung: Ergebnisse des Bertelsmann-Gesundheitsmonitors [Structural Differences between Health Insurance Funds and their Impact on Health Services Research: Results from the Bertelsmann Health-Care Monitor]. Das Gesundheitswesen 2012; 74: 291-297
[4] Hoffmann F, Icks A. Diabetes 'Epidemic' in Germany? A Critical Look at Health Insurance Data Sources. Experimental and Clinical Endocrinology \& Diabetes 2012; 120: 410-415

[5] Ramos AL, Hoffmann F. Differences in chronic back pain and joint disorders among health insurance funds. Results of a cross-sectional study based on the data of the Socioeconomic Panel from 2013. Zeitschrift Fur Rheumatologie 2017; 76: 238-244

[6] Jaunzeme J, Eberhard S, Geyer S. Wie „repräsentativ" sind GKV-Daten? Demografische und soziale Unterschiede und Ähnlichkeiten zwischen einer GKV-Versichertenpopulation, der Bevölkerung Niedersachsens sowie der Bundesrepublik am Beispiel der AOK Niedersachsen [How "representative" are data from statutory health insurances? Demographic and social differences and similarities between a statutory health insurance population, the population of Lower Saxony and the Federal Republic of Germany at the example of the AOK Niedersachsen]. Bundesgesundheitsblatt 2013; 56: 447-454

[7] Bundesagentur_fuer_Arbeit. Klassufikation der Berufe 2021. https:// statistik.arbeitsagentur.de/DE/Navigation/Grundlagen/Klassifikationen/Klassifikation-der-Berufe/KldB2010/KIdB2010-Nav.html. Zugegriffen: 29.01.2021

[8] Statistisches_Bundesamt. Bevölkerung: Bundesländer, Stichtag, Geschlecht, Altersjahre. Ergebnisse auf Grundlage des Zensus 2011: DESTATIS; 2019. https://www-genesis.destatis.de/genesis// online? operation $=$ table $\&$ code $=12411-0013 \&$ by pass $=$ true \&levelindex $=1$ \&levelid $=1596721224110$ \#abreadcrumb. Zugegriffen: 05.12.2019

[9] Bundesagentur_für_Arbeit. Beschäftigte nach Berufen (KldB 2010) (Quartalszahlen) Nürnberg: Bundesagentur_fuer_Arbeit; 2018. https://statistik.arbeitsagentur.de/Navigation/Statistik/Statistik-nachThemen/Statistik-nach-Themen-Nav.html. Zugegriffen: 11.10.2019

[10] Verordnung über die Erfassung und Übermittlung von Daten für die Träger der Sozialversicherung (Datenerfassungs-und-übermittlungsverordnung-in der Fassung der Bekanntmachung vom 23. Januar 2006 (BGBI. I S. 152), die zuletzt durch Artikel 27 des Gesetzes vom 4. August 2019 (BGBI. I S. 1147) geändert worden ist) [Decree about collection and transmission of data for the provider of social security in Germany; last amendment on 4th of August 2019], (2019)

[11] Bundesagentur_fuer_Arbeit. Meldeverfahren zur Sozialversicherung 2021. https://www.arbeitsagentur.de/betriebsnummern-service/ meldeverfahren-sozialversicherung. Zugegriffen: 30.01.2021

[12] Bundesagentur_fuer_Arbeit (2019). Meldeverfahren für Sozialversicherung. Schlüsselverzeichnis für die Angaben zur Tätigkeit. Bundesagentur_fuer_Arbeit, editor. Nürnberg

[13] Bundesagentur_für_Arbeit (2011). Klassifikation der Berufe 2010 (KLDB 2010). Nürnberg: Bundesagentur_für_Arbeit

[14] Weber G. Wechseldynamik, Wechslerprofile und Motive der Kassenwahlentscheidung. Empirische Befunde zum Konsumverhalten in der gesetzlichen Krankenversicherung. Gesundheits-und Sozialpolitik 2009; 6: 32-42

[15] Karasek R, Theorell T. Healthy work. New York: Basic Books.1990

[16] Murdock KW, LeRoy AS, Lacourt TE, Duke DC, Heijnen C], Fagundes CP. Executive functioning and diabetes: The role of anxious arousal and inflammation. Psychoneuroendocrinology 2016; 71: 102-109

[17] Steinvil A, Shirom A, Melamed S et al. Relation of Educational Level to Inflammation-Sensitive Biomarker Level. American Journal of Cardiology 2008; 102: 1034-1039

[18] Safieddine B, Sperlich S, Beller ] et al. Socioeconomic inequalities in type 2 diabetes among different population subgroups. European Journal of Public Health 2020; 30

[19] Tetzlaff J, Epping J, Sperlich S, Eberhard S, Stahmeyer JT, Geyer S. Widening inequalities in multimorbidity? Time trends among the working population between 2005 and 2015 based on German health insurance data. International Journal for Equity in Health 2018; 17: 103 
[20] Epping J, Muschik D, Geyer S. Social inequalities in the utilization of outpatient psychotherapy: analyses of registry data from German statutory health insurance. International Journal for Equity in Health 2017; 16: 147

[21] Glickmann L, Hubbard M, Liveright T, Valciukas A. Fall-off in reporting life events: Effects of life change, desirability, and anticipation. Behavioral Medicine 1990; 16: 31-37

[22] Candido E, Kurdyak P, Alter DA. Item nonresponse to psychosocial questionnaires was associated with higher mortality after acute myocardial infarction. Journal of Clinical Epidemiology 2011; 64: 213-222

[23] Cohen G, Duffy JC. Are nonrespondents to health surveys less healthy than respondents? Journal of Official Statistics 2002; 18: 13-24

[24] Tourangeau R, Rips LJ, Rasinski K. The Psychology of Survey Response. Cambridge: Cambridge University Press; 2000; 2000
[25] Walker M, Whincup PH, Shaper G, Lennon LT, Thomson A. Validation of Patient Recall of Doctor-diagnosed Heart Attack and Stroke: A Postel Questionnaire and Record Review Comparison. American Journal of Epidemiology 1998; 148: 355-361

[26] Bundesinstitut_für_Arzneimittel_und_Medizinprodukte. Informationssystem Versorgungsdaten 2020. https://www.dimdi.de/dynamic/de/ weitere-fachdienste/versorgungsdaten/. Zugegriffen: 13.07.2020

[27] Bundesinstitut_für_Arzneimittel_und_Medizinprodukte. Forschungsdatenzentrum (DaTraV) 2021. https://www.dimdi.de/dynamic/de/ weitere-fachdienste/forschungsdatenzentrum/. Zugegriffen: 30.1.2021

[28] Datentransparenzverordnung vom 19. Juni 2020 (BGBI. I S. 1371), (2020) 